\title{
Maximum incisal opening, caries experience and health-related quality of life in patients undergoing treatment for head and neck cancer - A longitudinal cohort study.
}

\section{Kanchan P Dholam}

Tata Memorial Hospital

\section{Priyanka Somani}

Tata Memorial Centre

Jinesh Dugad

Asian Cancer Institute

\section{Pankaj Kharade}

Aligarh Muslim University

Pallavi P Rane

Tata Memorial Centre

\section{Jai P Agarwal}

Tata Memorial Centre

Gurkaran Preet Singh ( $\nabla$ gurkaranpreetsingh@gmail.com )

Tata Memorial Centre https://orcid.org/0000-0002-1955-2783

\section{Research Article}

Keywords: trismus, head and neck cancer (HNC), maximal incisal opening (MIO), multimodal treatment, tumor sub-site

Posted Date: August 26th, 2021

DOl: https://doi.org/10.21203/rs.3.rs-553668/v1

License: (c) (1) This work is licensed under a Creative Commons Attribution 4.0 International License. Read Full License 


\section{Abstract \\ Purpose}

Prospective evaluation of the change in maximum incisal opening (MIO) in patients receiving treatment for head neck cancer (HNC), and its interaction with clinical parameters such as tumor site/ sub-site and type of treatment received. Secondary aim was to ascertain the longitudinal change in dental caries experience and health-related quality of life (HRQoL) during the study period.

\section{Methods}

Seventy treatment-naïve patients undergoing treatment for squamous cell carcinoma (SCC) of oral cavity and/or oropharynx were assessed at 3 designated time-points; pre-treatment $\left(T_{0}\right)$, immediate post-treatment $\left(T_{1}\right)$ and 6 months post-treatment $\left(T_{2}\right)$. The primary study variable was change in $\mathrm{MIO}(\mathrm{cm})$ across 3 time points $\left(T_{0}, T_{1}\right.$, $\mathrm{T}_{2}$ ). Dental caries incidence was measured using decayed, missing, filled teeth (DMFT) index and HRQoL was assessed using the European Organization for Research and Treatment of Cancer Quality of Life Questionnaires (EORTC QLQ-C30 and H\&N-35). Change in DMFT and HRQoL was assessed between $T_{0}$ and $T_{2}$. Non-parametric repeated measures ANOVA was used to analyse the longitudinal change in MIO and DMFT scores and their interaction with clinical parameters respectively. Wilcoxon signed-rank test was used to compare corresponding HRQoL domains between $T_{0}$ and $T_{2}$. All statistical tests were 2-sided, and differences with a $p$-value $<0.05$ were considered statistically significant.

\section{Results}

Sixty-seven eligible HNC patients showed a significant change in mouth-opening and dental caries experience from $T_{0}$ to $T_{2}$, irrespective of tumor site/ sub-site and type of treatment $(p<0.001)$. Site-wise, patients with oral cavity cancer and specifically maxillary tumors; treatment-wise those undergoing surgery and/or, multi-modal treatment showed persistently reduced mouth opening late post-treatment. There was a statistical increase in head-and-neck site specific HRQoL symptoms at $\mathrm{T}_{2}$. A high prevalence $(69 \%)$ of post-treatment trismus was noted especially in patients undergoing multi-modality treatment $(83 \%)$.

\section{Conclusion}

Patients undergoing treatment for HNC demonstrate a significant longitudinal change in mouth-opening and caries incidence. They may show partial recovery of $\mathrm{MIO}$ at 6-months after an initial decrease in the immediate post-treatment period.

\section{Introduction}

Trismus is a common treatment-related effect experienced by patients undergoing treatment for head and neck cancer (HNC). Surgical ablation of HNC may lead to fibrosis and scar contracture, which may impede optimal 
mouth opening. Patients who receive radiotherapy (RT) in definite or adjuvant setting with/without chemotherapy also report varying degrees of trismus [1].

The wide variance (39\% - 79\%) in prevalence of trismus amongst patients treated for HNC may be attributed to heterogeneity seen in site and stage of tumor, treatment modalities, differing sample sizes, follow-up periods and lack of uniform criteria for definition of trismus [1-8]. As proposed by Djikstra et al., maximal incisal opening (MIO) of $\leq 35 \mathrm{~mm}$ has now been accepted as indicative of trismus [2].

Trismus is known to have a significant impact on patient's daily activities such as chewing, swallowing and speech resulting in impairment of health-related quality of life (HRQoL) [9]. Difficulty in maintaining optimal oral hygiene can contribute to the development of dental caries and other serious odontogenic infections [10]. Majority of the published literature is on prevalence and predictive risk factors associated with trismus, derived from retrospective and/ or, cross-sectional studies with HNC patients predominantly undergoing single treatment modality, either primary radiotherapy or primary surgery [4-6, 11-16]. Literature on longitudinal change in $\mathrm{MIO}$ in relation to tumor site and treatment modality is sparse. Also, few studies have addressed the impact of mouth opening on quality of life (QoL), and these have yielded inconsistent findings [17].

The primary aim of the current study was to prospectively evaluate the change in maximum incisal opening (MIO) in patients receiving treatment for $\mathrm{HNC}$, from baseline to six months post-treatment completion, in relation to type of treatment received (surgery/ radiotherapy/ surgery + radiotherapy) and primary site/ sub-site of lesion. Secondary aim was to ascertain the longitudinal change in dental caries experience and HRQoL during the study period.

\section{Materials And Methodology}

Patients diagnosed with HNC, reporting to a tertiary care cancer center in India, were invited to participate in this longitudinal study. This prospective, observational study was initiated after obtaining approval from the Hospital Institutional Ethics Committee (HIEC). It was conducted in full accordance with ethical principles, including the World Medical Association Declaration of Helsinki (version 2002)

\section{Inclusion and exclusion criteria}

Consecutive, adult, treatment-naïve patients with biopsy-proven squamous cell carcinoma (SCC) of oral cavity and/or oropharynx were recruited after obtaining an informed consent. Patients received surgery ( \pm adjuvant radiotherapy) or definitive chemo-radiotherapy as per treatment-plan decided by a multi-disciplinary tumor board. Patients with recurrent HNC, carcinoma tongue treated with laser surgery, primary carcinoma of thyroid, larynx and skin were excluded. Patients reporting with trismus due to previous trauma, tetanus, temporo-mandibular disorder too were not included in the current study. Patients with completely edentulous single/both jaws or missing incisor teeth were also excluded to ensure homogeneity in reference points used to measure mouth opening.

\section{Participants and procedures}

As routine institutional practice, pre HNC-treatment, comprehensive assessment of dental health was done by a dental oncologist to eliminate oral foci of infection. Additionally, patients who received radiotherapy, whether in 
definite or adjuvant setting, underwent oral prophylaxis prior to initiation of therapy. In accordance with the

guidelines put forth by evidence-based management of cancers in India (2012-2013), patients received adjuvant radiotherapy 4 to 6 weeks after surgery based on the histological diagnosis of the resected specimen [18]. Information regarding patient's age, sex, tumor site and sub-site, TNM stage, treatment details (resection \pm reconstruction, radiotherapy, chemotherapy) was obtained from the electronic medical records. As routine practice, all patients were asked to follow a protocol of physical therapy one-week post-surgery or at the time of initiation of definitive radiotherapy. They were instructed to exercise active maximal mouth opening assisted by a jaw-stretching appliance. The regimen included 5 sessions per day of 5 opening/closing per session with 30 seconds stretch for each opening, as prescribed by an occupational onco-therapist.

\section{Assessments}

Patients, who consented to participate in the study, were assessed at the following 3 time-points,

1. Preoperative assessment (baseline) $-\mathrm{T}_{0}$

2. Immediate post-treatment assessment ( $2 \pm 1$ week after treatment completion) $-T_{1}$

3. Late post-treatment assessment ( 6 months \pm 2 weeks after treatment completion) $-T_{2}$

\section{Outcome measures}

(i) Evaluation of maximal incisal opening (MIO) at $\mathrm{T}_{0}, \mathrm{~T}_{1}$ and $\mathrm{T}_{2}$.

$\mathrm{MIO}(\mathrm{cm})$ was measured as the distance between incisal edges of corresponding opposing anterior teeth (central or lateral incisors) using a vernier caliper by a single operator. The patient was made to sit in an upright position and asked to open his/ her mouth as wide as possible. It was made sure that the same measurement point (either central or lateral incisor) was taken during each successive visit of the patient. In the current study, MIO measurements were done on a continuous scale to increase the chances of accurate results. This is in contrast to semi-quantitative method of grading of trismus used in majority of previously published literature. An average of two MIO measurements was taken at each visit of the patient.

(ii) Evaluation of dental caries incidence using dental caries index at $T_{0}$ and $T_{2}$.

Decayed, Missing, Filled teeth index (DMFT index) is a well-established key measure of caries experience in dental epidemiology [19]. The DMF index is applied to the permanent dentition and is expressed as the total number of teeth that are decayed (D), missing (M), or filled (F) in an individual.

(iii) Evaluation of health-related quality of life ( $\mathrm{HRQOL})$ at $\mathrm{T}_{0}$ and $\mathrm{T}_{2}$.

HRQoL was assessed using the European Organization for Research and Treatment of Cancer Quality of Life Questionnaire (EORTC QLQ-C30), and its head and neck module (EORTC QLQ H\&N-35). The raw data from the QoL scales were converted to scores ranging from 0 to 100 using linear transformation according to the standard scoring procedures. 
While MIO assessment was done at 3 time points $\left(T_{0}, T_{1}, T_{2}\right)$, DMFT and HRQoL assessments were carried out twice during the course of the current study, once at baseline $\left(T_{0}\right)$ and again at late post-treatment $\left(T_{2}\right)$ visits.

\section{Statistical Analyses}

Descriptive statistics were calculated according to standard procedures. Data analysis was carried out using SPSS (SPSS 21.0, IBM, NY, USA) and Npar LD package in R software (R 3.6.1). Interaction of longitudinal change in MIO and DMFT scores was statistically evaluated with the following clinical parameters:

a. Site of tumor (oral cavity/ oropharynx)

b. Oral cavity tumor sub-sites.

To ensure homogeneity, patients were grouped into the following oral cavity sub-sites:

(i) Buccal mucosa and mandible

(ii) Maxilla

(iii) Others (midline sites such as soft palate, tongue and lips)

c. Type of treatment received i.e., radical surgery (Sx) or definitive radiotherapy (RT) or surgery followed by adjuvant radiotherapy $(\mathrm{S} x+\mathrm{RT})$.

Due to non-normal distribution of data, it was reported and analysed as median with inter-quartile range (IQR) for continuous variables. Non-parametric repeated measures ANOVA was used to analyze the longitudinal change in MIO and DMFT scores over time and its interaction with each individual aforementioned clinical parameter respectively. MIO and DMFT scores between various clinical parameters at each time point were compared using Independent T test or Mann-Whitney $U$ test for 2 groups and ANOVA or Kruskal wallis test for more than 2 groups. Wilcoxon signed-rank test was used to compare corresponding HRQoL domains between baseline ( $\left.T_{0}\right)$ and late post-treatment $\left(T_{2}\right)$ visits. All statistical tests were 2-sided, and differences with a $p$-value $<0.05$ were considered to be statistically significant.

\section{Results}

A total of 70 patients (58 males; 12 females) undergoing treatment for oral or oropharyngeal SCC at our centre from January 2019 to July 2019, participated in the current study. The last patient to be recruited was followed up until January 2020. Forty-seven patients were diagnosed with oral cavity SCC while remaining twenty-three patients were diagnosed with carcinoma of oropharynx. The demographic and clinical characteristics of these patients, including details of tumor stage, site and sub-sites are listed in table 1.

Two-thirds of the cohort was diagnosed with advanced stage (T3-T4) lesions. Out of thirty-one patients who underwent surgical resection of tumor (with/without radiotherapy) (31/70; 44.3\%), seventeen patients underwent neck dissection at the time of surgery $(17 / 31 ; 54.8 \%)$. Majority of these surgically-treated patients received primary closure or did not undergo any other reconstructive procedure $(18 / 31 ; 58 \%)$. One-third of the surgical cohort $(11 / 31 ; 35.4 \%)$ underwent reconstruction with local/pedicled flaps or free flaps. In total, fifty-seven 
patients $(57 / 70,81.4 \%)$ received radiotherapy (primary/ adjuvant) to the head and neck region (mean dose - 64.7 \pm 5.87 , range $49-78 \mathrm{~Gy})$.

Sixty-seven patients $(67 / 70,95.7 \%)$ completed all the assessments at 3 designated time-points. Two patients $(2 / 70 ; 2.8 \%)$ expired during the follow-up period. One patient developed tumor recurrence which necessitated additional surgical procedure. Thus, 67 patients were eligible for prospective longitudinal analysis of change in MIO and DMFT scores in relation to clinical parameters (tumor site; sub-site; treatment modality), as specified in the objectives earlier. While change in MIO score was analyzed across 3 time points $\left(T_{0}, T_{1}, T_{2}\right)$, DMFT was assessed across $T_{0}$ and $T_{2}$. Pre-treatment $\left(T_{0}\right)$ HRQol scores were statistically compared with corresponding scores recorded at $6^{\text {th }}$ monthly visit $\left(\mathrm{T}_{2}\right)$ for all eligible 67 patients.

1. Longitudinal change in median MIO scores and relationship with clinical parameters

In the current study cohort, there was a statistically significant change in median MIO scores from baseline $\left(T_{0}\right)$ to 6-months post treatment $\left(T_{2}\right)$ across tumor sites, oral cavity sub-sites and treatment groups $(p<0.001)$ [figure 1 , figure 2, figure 3].

(i) Relationship between change in MIO score and tumor site or oral cavity tumor sub-site (figure 1, figure 2).

The change in median MIO score was significant between oral cavity and oropharyngeal cancers $(p<0.001)$. Pair-wise comparison revealed that patients with oropharyngeal cancers had significantly better mouth opening at all times $\left(T_{0} p=0.016 ; T_{1} p<0.001 ; T_{2} p=0.013\right)$. Additionally, patients with oral tumors $(p=0.001)$, and specifically maxillary tumors $(p=0.021)$, showed a statistically significant decrease in mouth opening 6-months post-treatment in comparison to corresponding baseline values.

(ii) Relationship between change in MIO score and treatment modality (figure 3)

There was no significant interaction between treatment modality and MIO score $(p=0.20)$. Amongst all patients, those who underwent combined modality treatment ( $\mathrm{S} x+\mathrm{RT}$ ) seem to fare worse (decreased mouth opening) when assessed across different time-points, although this interaction was not statistically significant $(p=0.57)$. However, pair-wise comparison showed that at $\mathrm{T}_{1}$, there was a statistically significant difference in median MIO score between treatment groups $(p=0.043)$. Patients who received adjuvant radiotherapy following surgery fared the worst (decreased mouth opening) at immediate post-treatment follow-up $\left(T_{1}\right)$. Subsequently, these patients who underwent multi-modality treatment showed a decrease in maximal mouth opening from baseline to 6months. However, it did not reach statistical significance $(p=0.173)$.

2. Longitudinal change in median DMFT scores and relationship with clinical parameters (table 2)

In the overall cohort, there was a statistically significant change in median DMFT scores from baseline $\left(T_{0}\right)$ to 6months post treatment $\left(T_{2}\right)$ across all treatment groups, tumor sites or oral cavity sub-sites $(p<0.001)$.

Pair-wise comparison showed a statistically significant difference in median DMFT scores between baseline $\left(T_{0}\right)$ and $T_{2}$, within patients who received definite radiotherapy $(p<0.001)$ or surgery followed by radiotherapy $(p=0.021)$. The same result was observed for patients undergoing treatment for either oral $(p<0.001)$ or oropharyngeal cancers $(p=0.003)$. Amongst oral cavity sub-sites, maxillary tumors $(p=0.016)$ and midline lesions 
$(p=0.03)$ showed a similar, statistically significant difference in median DMFT scores between baseline $\left(T_{0}\right)$ and $\mathrm{T}_{2}$.

\section{Prospective evaluation of HRQoL (table 3, table 4)}

There was a significant decrease in the scores of physical functioning (PF) $(p=0.00)$ and role of functioning (RF) $(p=0.006)$. There was an improvement in global QoL score, though it was not significant. There was a significant increase noted in fatigue score $(p=0.001)$ and loss of appetite $(p=0.001)$.

In addition to above, patients reported significant site-specific symptoms such as 'senses problem' $(p=0.00)$, 'teeth problems' $(p=0.005)$, 'dry mouth' $(p=0.00)$, 'opening of mouth' $(p=0.003)$ and 'sticky saliva' $(p=0.003)$ at 6months post treatment visit $\left(\mathrm{T}_{2}\right)$.

Additional analysis revealed that there was no statistical co-relation between MIO scores with q32 (Have you had pain in your jaw?) and q40 (Have you had problems opening your mouth wide?) of QLQ H\&N-35 questionnaire neither at $\mathrm{T}_{0}$ or $\mathrm{T}_{2}$ (table 5).

\section{Prevalence of trismus}

In our cohort, the overall prevalence of baseline trismus (tumor-burden trismus; $\mathrm{T}_{0}$ ) was $31.2 \%$. Trismus prevalence doubled in the immediate post-treatment period $\left(T_{1}\right)$ as it increased to $69 \%$. However, when measured at the late post-treatment visit $\left(\mathrm{T}_{2}\right)$, it decreased to $53.4 \%$. Interestingly, almost $3 / 4^{\text {th }}(73.3 \%)$ of the sub-cohort of patients diagnosed with trismus at baseline $\left(T_{0}\right)$ had persistent trismus even at 6 months late post-treatment visit $\left(\mathrm{T}_{2}\right)$. When analyzed based on treatment modality, the prevalence of trismus in the immediate posttreatment phase was highest in patients undergoing combined modality treatment i.e., surgery followed by adjuvant radiotherapy (83\%). It was slightly less in primary surgery group (78\%), while it was least in patients receiving definite radiotherapy $(59.4 \%)$.

\section{Discussion}

Trismus is a significant symptom experienced by HNC patients resulting in pain, dysphagia, malnutrition, thus complicating the clinical outcome. It affects the daily well-being of the patient resulting in a negative impact on overall quality of life. HRQoL results of the current study reflect the evidence published by Lee et al., Montalvo et al., Pauli et al., Lee et al. and Nagaraja et al. [3, 20-23]. Majority of cancer site-specific problems experienced by patients undergoing treatment for $\mathrm{HNC}$ can be regarded as sequalae of multi-modality treatment resulting in trismus, xerostomia and dental problems. However, in our study, MIO scores did not co-relate with specific questions in EORTC questionnaire describing problems related to jaw pain and/or, opening mouth wide. Patients did not perceive existing mouth opening as a problem affecting their day-to-day activities. This may be assumed to be the reason for lack of statistical difference in global quality of life score between two longitudinal timepoints $\left(T_{0}\right.$ and $\left.T_{2}\right)$.

Inability to maintain optimal oral hygiene, shift of oral flora in favor of cariogenic organisms, dietary changes along with radiotherapy-induced xerostomia puts the patient at a higher risk of developing dental caries [24]. Our results related to statistically significant increase in DMFT scores in HNC patients, especially those treated with 
radiotherapy (definite or adjuvant), corroborated the data published in a recent multi-centric study by Paglioni et al. [25]. They concluded that radiation-caries was associated with higher DMFT scores and lower HRQoL scores.

Much of the recent research has focused on understanding predictive risk factors associated with development of trismus in HNC patients $[7,14,16,26]$. In a cross-sectional study, Pantvaidya et al. identified clinical and sociodemographic risk factors for developing trismus [16]. Van der Geer et al. have proposed a predictive riskcalculation tool for treatment-naïve HNC patients to enable clinicians to take early, preventive steps [14]. However due to cross-sectional design, these studies had largely heterogenous data-sets and did not report on clinical course of trismus over time. There have been only four prospective studies on trismus in HNC patients published in literature so far [3, 4, 27-29]. While Wetzel et al. evaluated prospective changes in maximal mouth opening in oral cancer patients, Wang et al. studied degree and time-course changes of radiation-induced trismus occurring after radiotherapy for nasopharyngeal cancer $[27,29]$. However, unlike our study, the former study had inadequate representation of patients receiving definite radiotherapy and latter included patients receiving radiotherapy exclusively. Studies by Lee et al. ${ }^{3}$ and Scott et al. ${ }^{4}$ have focused on prediction of trismus after treatment in HNC patients [3, 4].

One of the strengths of the current study is prospective measurement of maximal incisal opening (MIO) in HNC patients, stratified homogenously according to tumor site/ sub-site and type of treatment received. This enabled us to accurately analyze effect of distinct tumor sites on longitudinal change in MIO after single-/ multi-modality treatment. Also, majority of previous studies have reported on trismus using a dichotomous scale (cut-off point $35 \mathrm{~mm}$ ). In our study, MIO was measured on a continuous scale to increase chances the accurate results.

The current study revealed that patients with HNC experience a significant change in MIO scores from pretreatment stage to 6 months post-treatment, irrespective of the site/ sub-site of lesion and type of treatment received. Generally, median MIO scores demonstrated a trend towards partial recovery at 6 -months. Previous studies by Lee et al., Scott et al., Wetzel et al. and others have reported a similar time-course of MIO in HNC patients $[3,4,7,27]$.

Our results demonstrated that patients receiving multi-modal treatment $(\mathrm{Sx}+\mathrm{RT})$ show significantly decreased mouth opening immediately post-treatment $\left(T_{1}\right)$ when compared with those receiving single modality treatment (Sx or RT). This difference in MIO scores remained the same even at 6-months post-treatment $\left(\mathrm{T}_{2}\right)$, although it did not reach statistical significance. Patients who received definite radiotherapy showed a better recovery in their ability to open mouth post-treatment $\left(T_{2}\right)$ as compared to patients who underwent surgery and/ or, multimodal treatment $(\mathrm{Sx}+\mathrm{RT})$. It may be valuable to note here that more than $90 \%$ of patients receiving definite radiotherapy were diagnosed with oropharyngeal tumors.

This is in contrast to the observations made by Wetzel et al. who demonstrated significantly higher mouth opening in the surgery group than in combined therapy $(\mathrm{Sx}+\mathrm{RT})$ or primary radiotherapy groups at both 6-month and 12-month follow-up visits [27]. Our results underline the importance of multi-modal treatment of oral cancer as a significant risk factor in the development of trismus, which has been noted previously by various investigators $[1,4,16,27,30]$.

In sharp contrast to the current study, Lee et al. in their prospective trial, found that post-operative radiotherapy ( \pm chemotherapy) and primary tumor site did not predict the development of trismus [3]. Site of lesion was 
observed to have profound effect on maximal mouth opening of HNC patients in our study. Oral tumors demonstrated significantly poorer outcome of mouth opening when compared to oropharynx lesions at pretreatment, immediate post-treatment and 6 months post treatment stages. This finding corroborates well with the results of Wetzel et al. and Astradsson et al., who concluded the same [27, 28].

In a recent paper, Pantvaidya et al. found oral cavity tumor sub-site to be predictor of trismus [16]. As a validation of the above, the current prospective study found that all 3 sub-sites within oral cavity demonstrated similar effect of decrease in mouth opening from pre-treatment to late post-treatment. Similar to results published by Wetzel et al., we found smaller pre-treatment mouth-opening in patients diagnosed with mandible-buccal mucosa lesions [27]. Although, unlike their study, the difference in pre-treatment MIO scores amongst 3 sub-sites was not statistically significant. Furthermore, patients with midline and/or, mandible/ buccal mucosa lesions showed a partial recovery in mouth opening by 6 months. However, patients with maxillary lesions demonstrated a statistically significantly reduced MIO at 6 months when compared with pre-treatment levels.

In general, it can be stated that tumors located near the temporomandibular joint and masticatory muscles will increase the risk of developing trismus due to infiltration/ irritation and post-treatment scarring/ fibrosis [6, 26. $27,30,31]$. Also, patients with posterior maxillary/ mandibular tumors or buccal mucosal lesions show smaller mouth opening compared to anteriorly placed lesions [27].

There was a high prevalence $(69 \%)$ of post-treatment trismus in our study especially in those patients undergoing multi-modality treatment $(83 \%)$ for cancer (surgery + radiotherapy \pm chemotherapy). Although it is marginally higher than prevalence rate mentioned by Wetzel et al. and Scott et al. it falls within the wide range of prevalence documented in literature [4, 27]. It is important to note that, in the pre-treatment stage, nearly one-third of cohort (31.2\%) already had reduced mouth opening. As mentioned by Pantvaidya et al., pre-existing submucous fibrosis is widely prevalent in our country which can contribute significantly to development of posttreatment trismus [15]. Also, majority (64\%) of our patients were diagnosed with advanced T stage lesions (T3, T4) which necessitated extensive resection followed by adjuvant therapy, thereby resulting in combined harmful effects of treatment. In the current study, almost $50 \%$ of the cohort experienced trismus at the late 6-months post-treatment visit. Our data is in concurrence with Wang et al. ${ }^{29}$, who observed that trismus evolves rapidly during the first nine months after HNC treatment [29].

The current study gives an insight into the time-course relationship of maximal mouth opening in newlydiagnosed patients undergoing treatment for HNC. Need for early institution of appropriate physiotherapy regimens in HNC patients may be argued here. But patient's compliance becomes an integral issue for it to be successful. A recent prospective study reported favorable effects of early, structured jaw-exercise therapy on MIO and HRQoL in HNC patients [32]. Nonetheless, future research should focus on correctly identifying patients who are at a high risk for development of trismus before initiation of oncologic therapy. Perhaps, as suggested by van der Geer et al., a risk-prediction tool may be used to identify and recruit such high-risk patients to study effectiveness of various jaw-stretching exercises/ therapies [14].

\section{Conclusion}

Patients with head and neck cancer experience a significant change in mouth-opening from baseline $\left(T_{0}\right)$ to 6 months post-treatment $\left(\mathrm{T}_{2}\right)$, irrespective of the site/ sub-site of lesion and type of treatment received. They may 
show partial recovery at 6-months $\left(T_{2}\right)$ after an initial decrease in the immediate post-treatment period $\left(T_{1}\right)$. Patients with oral cavity cancer and specifically maxillary tumors continue to show significantly reduced mouth opening up to 6 months after treatment. Treatment-wise patients undergoing surgery and/or, multi-modal treatment may exhibit a trend to show persistently reduced mouth opening late post-treatment.

\section{Declarations}

1. Funding: This research did not receive any specific grant from funding agencies in the public, commercial, or not-for-profit sectors.

2. Conflicts of interest/Competing interests: The authors have no conflicts of interest to declare that are relevant to the content of this article.

3. Availability of data and material: Available on request

4. Code availability: N/A

\section{Authors' contributions}

Conception and design or analysis and interpretation of data: Kanchan Dholam, Priyanka Somani, Gurkaran Preet Singh, Jai Prakash Agarwal, Jinesh Dugad, Pankaj Kharade, Pallavi Rane

Drafting of the manuscript or revising it for important intellectual content: Kanchan Dholam, Gurkaran Preet Singh.

Final approval of the version to be published: Kanchan Dholam, Gurkaran Preet Singh.

6. Ethics approval: This prospective, observational study was initiated after obtaining approval from the Hospital Institutional Ethics Committee (HIEC). It was conducted in full accordance with ethical principles, including the World Medical Association Declaration of Helsinki (version 2002)

7. Consent to participate: Informed consent was obtained from all individual participants included in the study.

8. Consent for publication: The authors affirm that human research participants provided informed consent for publication of the data collected during the study.

\section{Author declaration:}

The work submitted here has not been published, that it is not under consideration for publication elsewhere, that its publication is approved by all authors and tacitly or explicitly by the responsible authorities where the work was carried out, and that, if accepted, it will not be published elsewhere in the same form, in English or in any other language, including electronically without the written consent of the copyright-holder.

\section{References}


1. Dijkstra PU, Kalk WWI, Roodenburg JLN (2004) Trismus in head and neck oncology: A systematic review. Oral Oncol 40:879-889. doi:10.1016/j.oraloncology.2004.04.003

2. Dijkstra PU, Huisman PM, Roodenburg JL (2006) Criteria for trismus in head and neck oncology. Int J Oral Maxillofac Surg 35:337-342. doi:10.1016/j.ijom.2005.08.001

3. Lee R, Slevin N, Musgrove B, Swindell R, Molassiotis A (2012) Prediction of post-treatment trismus in head and neck cancer patients. Br J Oral Maxillofac Surg 50:328-332. doi:10.1016/j.bjoms.2011.06.009

4. Scott B, D'Souza J, Perinparajah N, Lowe D, Rogers SN (2011) Longitudinal evaluation of restricted mouth opening (trismus) in patients following primary surgery for oral and oropharyngeal squamous cell carcinoma. Br J Oral Maxillofac Surg 49:106-111. doi:10.1016/j.bjoms.2010.02.008

5. Louise Kent M, Brennan MT, Noll JL et al (2008) Radiation-induced trismus in head and neck cancer patients. Support Care in Cancer 16:305-309. doi:10.1007/s00520-007-0345-5

6. Kamstra JI, Dijkstra PU, van Leeuwen M, Roodenburg JL, Langendijk JA (2015) Mouth opening in patients irradiated for head and neck cancer: a prospective repeated measures study. Oral Oncol 51:548-555. doi:10.1016/j.oraloncology.2015.01.016

7. van der Geer SJ, Kamstra Jl, Roodenburg JL et al (2016) Predictors for trismus in patients receiving radiotherapy. Acta Oncol 55:1318-1323. doi:10.1080/0284186X.2016.1223341

8. Loh SY, Mcleod RWJ, Elhassan HA (2017) Trismus following different treatment modalities for head and neck cancer: a systematic review of subjective measures. Eur Arch Otorhinolaryngol 274:2695-2707. doi:10.1007/s00405-017-4519-6

9. Johnson J, Johansson M, Rydén A, Houltz E, Finizia C (2015) Impact of trismus on health-related quality of life and mental health. Head Neck 37:1672-1679. doi:10.1002/hed.23816

10. Martins CA, Goldenberg DC, Narikawa R, Kowalski LP (2020) Trismus and oral health conditions during diagnosis of malignant oral neoplasms. Braz J Otorhinolaryngol 86:552-557.

doi:10.1016/j.bjorl.2019.02.004

11. Van Cann EM, Dom M, Koole R, Merkx MA, Stoelinga PJ (2005) Health related quality of life after mandibular resection for oral and oropharyngeal squamous cell carcinoma. Oral Oncol 41:687-693. doi:10.1016/j.oraloncology.2005.03.001

12. Chen YY, Zhao C, Wang J et al (2011) Intensity-modulated radiation therapy reduces radiation-induced trismus in patients with nasopharyngeal carcinoma: a prospective study with $>5$ years of follow-up. Cancer 117:2910-2916. doi:10.1002/cncr.25773

13. Lindblom U, Garskog O, Kjellen E et al (2014) Radiation-induced trismus in the ARTSCAN head and neck trial. Acta Oncol 53:620-627. doi:10.3109/0284186X.2014.892209

14. van der Geer SJ, van Rijn PV, Kamstra JI et al (2019) Prevalence and prediction of trismus in patients with head and neck cancer: A cross-sectional study. Head Neck 41:64-71. doi:10.1002/hed.25369

15. Hague C, Beasley W, Garcez K et al (2018) Prospective evaluation of relationships between radiotherapy dose to masticatory apparatus and trismus. Acta Oncol 57:1038-1042.

doi:10.1080/0284186X.2018.1459047

16. Pantvaidya G, Sivasanker M, Ranganathan P, Pai P, D'Cruz A (2019) Prospective cross-sectional study assessing prevalence and factors affecting trismus after multimodal treatment for oral cancers. Head Neck 41:286-290. doi:10.1002/hed.25464

Page $11 / 24$ 
17. Steiner F, Evans J, Marsh R, Rigby P, James S, Sutherland K et al (2015) Mouth opening and trismus in patients undergoing curative treatment for head and neck cancer. Int J Oral Maxillofac Surg 44:292-296. doi:10.1016/j.ijom.2014.12.009

18. Evidence Based Management of Cancers in India (2012) -13). Guidelines for Head and Neck Cancers. XI Part A. Published by Tata Memorial Centre, Mumbai

19. Moradi G, Mohamadi Bolbanabad A, Moinafshar A, Adabi H, Sharafi M, Zareie B (2019) Evaluation of Oral Health Status Based on the Decayed, Missing and Filled Teeth (DMFT) Index. Iran J Public Health 48:20502057. PMID: 31970104; PMCID: PMC6961190

20. Montalvo C, Finizia C, Pauli N, Fagerberg-Mohlin B, Andréll P (2020) Impact of exercise with TheraBite device on trismus and health-related quality of life: A prospective study. Ear Nose Throat J 9:145561320961727. doi:10.1177/0145561320961727

21. Pauli N, Fagerberg-Mohlin B, Andréll P, Finizia C (2014) Exercise intervention for the treatment of trismus in head and neck cancer. Acta Oncol 53:502-509. doi:10.3109/0284186X.2013.837583

22. Lee LY, Chen SC, Chen WC, Huang BS, Lin CY (2015) Postradiation trismus and its impact on quality of life in patients with head and neck cancer. Oral Surg Oral Med Oral Pathol Oral Radiol 119:187-195.

doi:10.1016/j.oooo.2014.10.003

23. Nagaraja S, Kadam SA, Selvaraj K, Ahmed I, Javarappa R (2016) Trismus in head and neck cancer patients treated by telecobalt and effect of early rehabilitation measures. J Cancer Res Ther 12:685-688. doi:10.4103/0973-1482.176181

24. Pow EH, McMillan AS, Leung WK, Kwong DL, Wong MC (2003) Oral health condition in southern Chinese after radiotherapy for nasopharyngeal carcinoma: extent and nature of the problem. Oral Dis 9:196-202. doi:10.1034/j.1601-0825.2003.02924.x

25. de Pauli Paglioni M, Palmier NR, Prado-Ribeiro AC, Fregnani ER, Gavião MBD, Brandão TB et al (2020) The impact of radiation caries in the quality of life of head and neck cancer patients. Support Care Cancer 28:2977-2984. doi:10.1007/s00520-019-05171-8

26. Weber C, Dommerich S, Pau HW, Kramp B (2010) Limited mouth opening after primary therapy of head and neck cancer. Oral Maxillofac Surg 14:169-173. doi:10.1007/s10006-010-0220-2

27. Wetzels JW, Merkx MA, de Haan AF, Koole R, Speksnijder CM (2014) Maximum mouth opening and trismus in 143 patients treated for oral cancer: a 1-year prospective study. Head Neck 36:1754-1762. doi:10.1002/hed.23534

28. Astradsson T, Laurell G, Ahlberg A, Nikolaidis P, Johansson H, Ehrsson YT (2018) Trismus in patients with head and neck cancer and 5-year overall survival. Acta Otolaryngol 138:1123-1127.

doi:10.1080/00016489.2018.1511059

29. Wang CJ, Huang EY, Hsu HC, Chen HC, Fang FM, Hsiung CY (2005) The degree and time-course assessment of radiation-induced trismus occurring after radiotherapy for nasopharyngeal cancer. Laryngoscope 115:1458-1460. doi:10.1097/01.mlg.0000171019.80351

30. Goldstein M, Maxymiw WG, Cummings BJ, Wood RE (1999) The effects of antitumor irradiation on mandibular opening and mobility: a prospective study of 58 patients. Oral Surg Oral Med Oral Pathol Oral Radiol Endod 88:365-373. doi:10.1016/s1079-2104(99)70044-2 
31. Ichimura K, Tanaka T (1993) Trismus in patients with malignant tumours in the head and neck. J Laryngol Otol 107:1017-1020. doi:10.1017/s0022215100125149

32. Karlsson O, Karlsson T, Pauli N, Andréll P, Finizia C (2020) Jaw exercise therapy for the treatment of trismus in head and neck Cancer: a prospective three-year follow-up study. Support Care Cancer. https://doi.org/10.1007/s00520-020-05517-7

\section{Tables}

\section{Table 1}

Demographic and clinical details of the study cohort $(n=70)$ 


\begin{tabular}{|c|c|c|}
\hline S No. & Patient characteristics & Number $(n=70)$ \\
\hline \multirow[t]{3}{*}{01.} & Gender & \\
\hline & Male & 58 \\
\hline & Female & 12 \\
\hline 02. & Mean age at presentation & 53 years \\
\hline \multirow[t]{5}{*}{03.} & Tumor site/ sub-sites & \\
\hline & Oral cavity & 47 \\
\hline & Buccal mucosa and/or, mandible lesions & 17 \\
\hline & Maxillary lesions & 14 \\
\hline & Midline lesions & 16 \\
\hline & Oropharynx & 23 \\
\hline & Glottis & 5 \\
\hline & Pyriform fossa & 9 \\
\hline & Tonsil & 7 \\
\hline & Posterior pharyngeal wall & 2 \\
\hline
\end{tabular}

04. T stage

T1

T2

T3

T4

Tx
7

14

18

28

3

\section{Treatment received}

Radiotherapy ( \pm Concurrent chemotherapy) 39

Surgery

Surgery alone

Surgery + Radiotherapy ( \pm Chemotherapy) 18 
06. Radiation details $(n=57)$

$\geq 60 \mathrm{~Gy} / 30 \# \quad 48$

$<60 \mathrm{~Gy} / 30 \# \quad 9$

Mean radiation dose $( \pm$ s.d $) \quad 64.7( \pm 5.87)$

07. Surgery details $(n=31)$

a. Neck dissection

$\begin{array}{ll}\text { Yes } & 17\end{array}$

No $\quad 53 / 70,14 / 31$

b. Reconstruction details $(n=31)$

\begin{tabular}{ll}
\hline Primary closure/ No reconstruction & 18 \\
\hline Split-skin grafts & 2 \\
\hline Free flaps & 3 \\
\hline Local/ Pedicle flaps & 8 \\
\hline
\end{tabular}




\section{Table 2}

Results of non-parametric repeated measures ANOVA used to analyze longitudinal change in DMFT index score over time $\left(T_{0}, T_{2}\right)$ and its interaction with clinical parameters $(n=65)$. Inter-group pair-wise comparison at each time-point was done using independent $T$ test or Mann-Whitney $U$ test for 2 groups and ANOVA or Kruskal Wallis test for more than 2 groups. Intra-group pair-wise analysis between baseline $\left(T_{0}\right)$ and 6-months posttreatment $\left(\mathrm{T}_{2}\right)$ visits was done using Wilcoxon-signed rank test. Differences with a $\mathrm{p}$-value $<0.05$ considered statistically significant. 


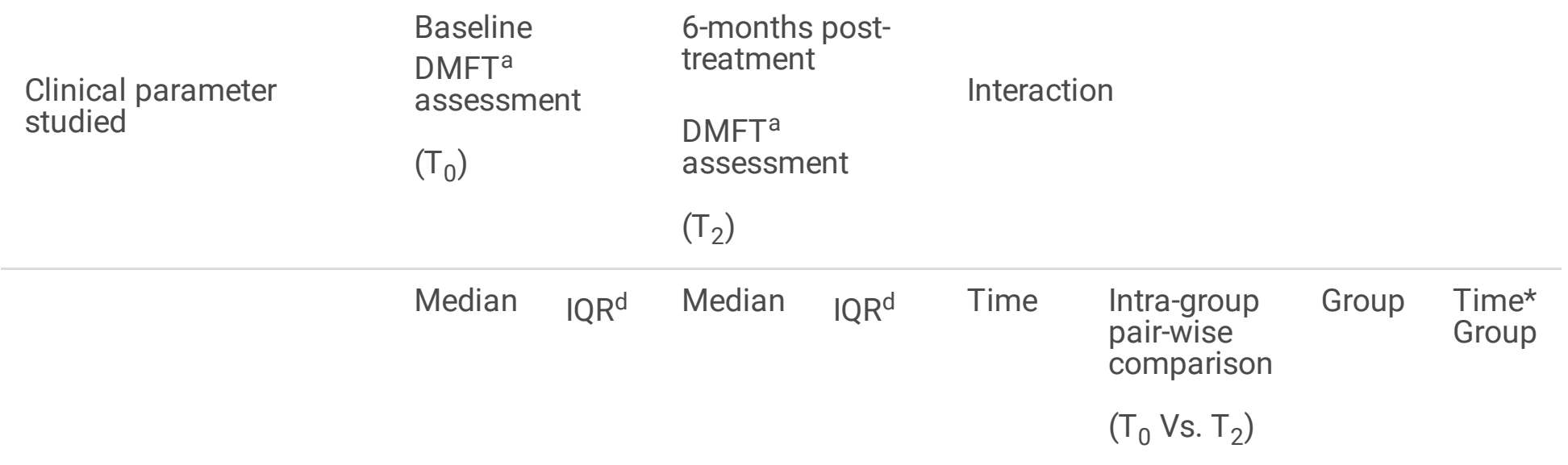

\section{Tumor \\ sites}

\begin{tabular}{|c|c|c|c|c|c|c|c|c|}
\hline Oral Cavity & 2.000 & $\begin{array}{l}0.25- \\
5\end{array}$ & 6.000 & $\begin{array}{l}2.625- \\
9.75\end{array}$ & $<0.001$ & $<0.001$ & 0.98 & 0.17 \\
\hline Oropharynx & 3.500 & $\begin{array}{l}0- \\
8.25\end{array}$ & 5.500 & $\begin{array}{l}0.75- \\
10.25\end{array}$ & & 0.003 & & \\
\hline
\end{tabular}

Oral

cavity

tumor

sub-sites

\begin{tabular}{|c|c|c|c|c|c|c|c|}
\hline $\begin{array}{l}\text { Buccal } \\
\text { Mucosa- }\end{array}$ & 3.50 & $\begin{array}{l}0.75- \\
4\end{array}$ & 10.0 & $\begin{array}{l}4.5- \\
11.75\end{array}$ & $<0.001$ & & 0.04 \\
\hline Mandible & & & & & & 0.144 & \\
\hline Maxilla & 2.00 & $\begin{array}{l}1- \\
5.75\end{array}$ & 6.500 & $\begin{array}{l}3- \\
11.75\end{array}$ & & 0.016 & \\
\hline $\begin{array}{l}\text { Midline } \\
\text { lesions }\end{array}$ & 5.00 & $1-6$ & 7.000 & $\begin{array}{l}2.25- \\
9.5\end{array}$ & & 0.03 & \\
\hline
\end{tabular}

\section{Type of}

treatment

\begin{tabular}{|c|c|c|c|c|c|c|c|c|}
\hline $\mathrm{RT}^{\mathrm{b}}$ alone & 3.00 & $\begin{array}{l}0- \\
6.75\end{array}$ & 5.000 & $\begin{array}{l}0.25- \\
9.75\end{array}$ & $<0.001$ & $<0.001$ & 0.44 & 0.47 \\
\hline $\begin{array}{l}\text { Surgery } \\
\text { alone }\end{array}$ & 1.50 & $\begin{array}{l}0.25- \\
4.75\end{array}$ & 5.500 & $\begin{array}{l}1.5- \\
8.5\end{array}$ & & 0.102 & & \\
\hline $\begin{array}{l}\text { Surgery } \\
+\mathrm{RT}^{\mathrm{C}}\end{array}$ & 2.00 & $2-5$ & 6.000 & 3-12 & & 0.021 & & \\
\hline
\end{tabular}

aDMFT- Decayed Missing Filled teeth

${ }^{\text {b RT }}$ alone- Radiotherapy alone

'Surgery + RT - Surgery followed by adjuvant radiotherapy 
$\mathrm{f}_{\mathrm{IQR}}$ - Inter-quartile range

\section{Table 3}

Results of Wilcoxon signed-rank test used to compare corresponding HRQoL domains in EORTC QLQ C-30 questionnaire, between baseline $\left(T_{0}\right)$ and 6-months post-treatment $\left(T_{2}\right)$ visits $(n=65)$. Differences with a $p$-value < 0.05 considered to be statistically significant.

\begin{tabular}{|c|c|c|c|}
\hline & Baseline HRQoL ${ }^{\mathrm{c}}$ assessment $\left(\mathrm{T}_{0}\right)$ & 6-months post-treatment & \\
\hline QLQ C-30 & Median (IQR)d & HRQoL ${ }^{C}$ assessment $\left(T_{2}\right)$ & $\mathrm{p}$-value \\
\hline Subscales ${ }^{b}$ & & Median (IQR) ${ }^{d}$ & \\
\hline PF & $93.33(80-100)$ & $86.67(66.67-93.33)$ & 0.000 \\
\hline $\mathrm{RF}$ & 100(83.33-100) & $83.33(66.67-100)$ & 0.006 \\
\hline EF & 75(58.33-91.67) & 75(58.33-91.67) & 0.090 \\
\hline $\mathrm{CF}$ & $83.33(66.67-100)$ & $91.67(66.67-100)$ & 0.903 \\
\hline SF & 83.33(66.67-100) & $83.33(66.67-100)$ & 0.674 \\
\hline QL & $58.33(50-77.08)$ & $66.67(50-85.42)$ & 0.555 \\
\hline FA & $22.22(11.11-33.33)$ & 33.33(11.11-44.44) & 0.001 \\
\hline NV & $0(0-0)$ & $0(0-16.67)$ & 0.052 \\
\hline PA & 16.67(0-33.33) & $16.67(0-33.33)$ & 0.099 \\
\hline SL & $0(0-33.33)$ & $0(0-33.33)$ & 0.378 \\
\hline AP & $0(0-33.33)$ & $33.33(0-58.33)$ & 0.001 \\
\hline $\mathrm{CO}$ & $0(0-33.33)$ & $0(0-33.33)$ & 0.268 \\
\hline $\mathrm{FI}$ & $33.33(0-66.67)$ & $33.33(0-66.67)$ & 0.443 \\
\hline
\end{tabular}


${ }^{a}$ QLQ C-30 - European Organization for Research and Treatment in Cancer Quality of Life Questionnaire Core $30,{ }^{C} \mathrm{HRQ}$ oL - Health-related quality of life, ${ }^{\mathrm{d}} \mathrm{QQR}$ - Interquartile range

bSub-scales: QL- Global health status/ QoL; Functional scales: PF - Physical functioning, RF - Role functioning, EF- Emotional functioning, CF- Cognitive functioning, SF- Social functioning; Symptom scales / items: FAFatigue, NV- Nausea and vomiting, PA- Pain, DY- Dyspnoea, SL- Insomnia, AP- Appetite loss, CO- Constipation, DIDiarrhoea, FI- Financial difficulties

\section{Table 4}

Results of Wilcoxon signed-rank test used to compare corresponding HRQoL domains in EORTC QLQ HN-35 questionnaire, between baseline $\left(T_{0}\right)$ and 6 -months post-treatment $\left(T_{2}\right)$ visits $(n=65)$. Differences with a $p$-value < 0.05 considered to be statistically significant. 


\begin{tabular}{|c|c|c|c|}
\hline & Baseline $\mathrm{HRQoL}^{\mathrm{C}}$ assessment $\left(\mathrm{T}_{0}\right)$ & 6-months post-treatment & \\
\hline QLQ HN-35ª & Median (IQR) ${ }^{\mathrm{d}}$ & HRQoL ${ }^{\mathrm{C}}$ assessment $\left(\mathrm{T}_{2}\right)$ & p-value \\
\hline Symptom scales ${ }^{b}$ & & Median (IQR) ${ }^{d}$ & \\
\hline HNPA & 16.67(8.33-33.33) & 23.61(2.08-41.67) & 0.402 \\
\hline HNSW & 8.33(0-16.67) & $16.67(0-25)$ & 0.274 \\
\hline HNSE & $0(0-16.67)$ & 16.67(0-33.33) & 0.000 \\
\hline HNSP & 11.11(0-30.56) & 19.44(11.11-22.22) & 0.039 \\
\hline HNSO & $8.33(0-25)$ & 16.67(0-41.67) & 0.012 \\
\hline HNSC & $0(0-13.33)$ & $6.67(0-20)$ & 0.126 \\
\hline HNSX & $0(0-33.33)$ & $0(0-33.33)$ & 0.527 \\
\hline HNTE & 33.33(0-33.33) & 33.33(0-66.67) & 0.005 \\
\hline HNOM & $0(0-33.33)$ & 33.33(0-58.33) & 0.003 \\
\hline HNDR & $0(0-33.33)$ & 33.33(0-66.67) & 0.000 \\
\hline HNSS & $0(0-33.33)$ & 33.33(0-66.67) & 0.003 \\
\hline HNCO & $0(0-33.33)$ & $0(0-33.33)$ & 0.586 \\
\hline HNFI & $0(0-33.33)$ & $0(0-33.33)$ & 0.461 \\
\hline HNPK & 100(0-100) & 100(0-100) & 0.394 \\
\hline HNNU & $0(0-100)$ & 100(0-100) & 0.071 \\
\hline HNFE & $0(0-100)$ & $0(0-100)$ & 0.248 \\
\hline HNWL & 100(0-100) & $0(0-100)$ & 0.223 \\
\hline HNWG & $0(0-100)$ & $0(0-100)$ & 0.782 \\
\hline
\end{tabular}


${ }^{a}$ QLQ HN-35 - European Organization for Research and Treatment in Cancer Quality of Life Questionnaire Head \& Neck module - 35, ${ }^{\mathrm{C}} \mathrm{HRQ}$ oL - Health-related quality of life, ${ }^{\mathrm{d}} \mathrm{Q} \mathrm{R}$ - Interquartile range

bSymptom scales: HNPA- Pain, HNSW- Swallowing, HNSE- Senses problems, HNSP- Speech problems, HNSOTrouble with social eating, HNSC- Trouble with social contact, HNSX- Less sexuality, HNTE- Teeth, HNOMOpening mouth, HNDR- Dry mouth, HNSS- Sticky saliva, HNCO- Coughing, HNFI- Felt ill, HNPK- Pain killers, HNNUNutritional supplements, HNFE- Feeding tube, HNWL- Weight loss, HNWG- Weight gain.

\section{Table 5}

Results of correlation analysis to check for correlation between q32 and q40 of EORTC HN-35 and $\mathrm{MIO}^{\mathrm{b}}$ scores at baseline $\left(T_{0}\right)$ and six-months follow-up visit $\left(T_{2}\right)$.

\begin{tabular}{|lll|}
\hline & $\begin{array}{l}\text { Baseline } \mathrm{MIO}^{\mathrm{b}} \\
\text { assessment }\left(\mathrm{T}_{0}\right) \\
\text { Spearman's rho ' } r \text { ' ( } \mathrm{p}\end{array}$ & $\begin{array}{l}\text { 6-months post-treatment } \mathrm{MIO} \mathrm{O}^{\mathrm{b}} \\
\text { assessment }\left(\mathrm{T}_{2}\right)\end{array}$ \\
value) & Spearman's rho ' $r$ ' ( $\mathrm{p}$ value $)$ \\
\hline Q32 - Have you had pain in your jaw? & $-0.192,(\mathrm{p}=0.12)$ & $-0.25,(\mathrm{p}=0.39)$ \\
\hline $\begin{array}{l}\text { Q40 - Have you had problems opening } \\
\text { your mouth wide? }\end{array}$ & $-0.157,(\mathrm{p}=0.2)$ & $-0.179,(\mathrm{p}=0.54)$ \\
\hline
\end{tabular}

${ }^{a}$ EORTC HN-35 - European Organization for Research and Treatment in Cancer Quality of Life Questionnaire Head \& Neck module - 35 .

${ }^{\mathrm{b}} \mathrm{MIO}$ - Maximal Incisal Opening

\section{Figures}




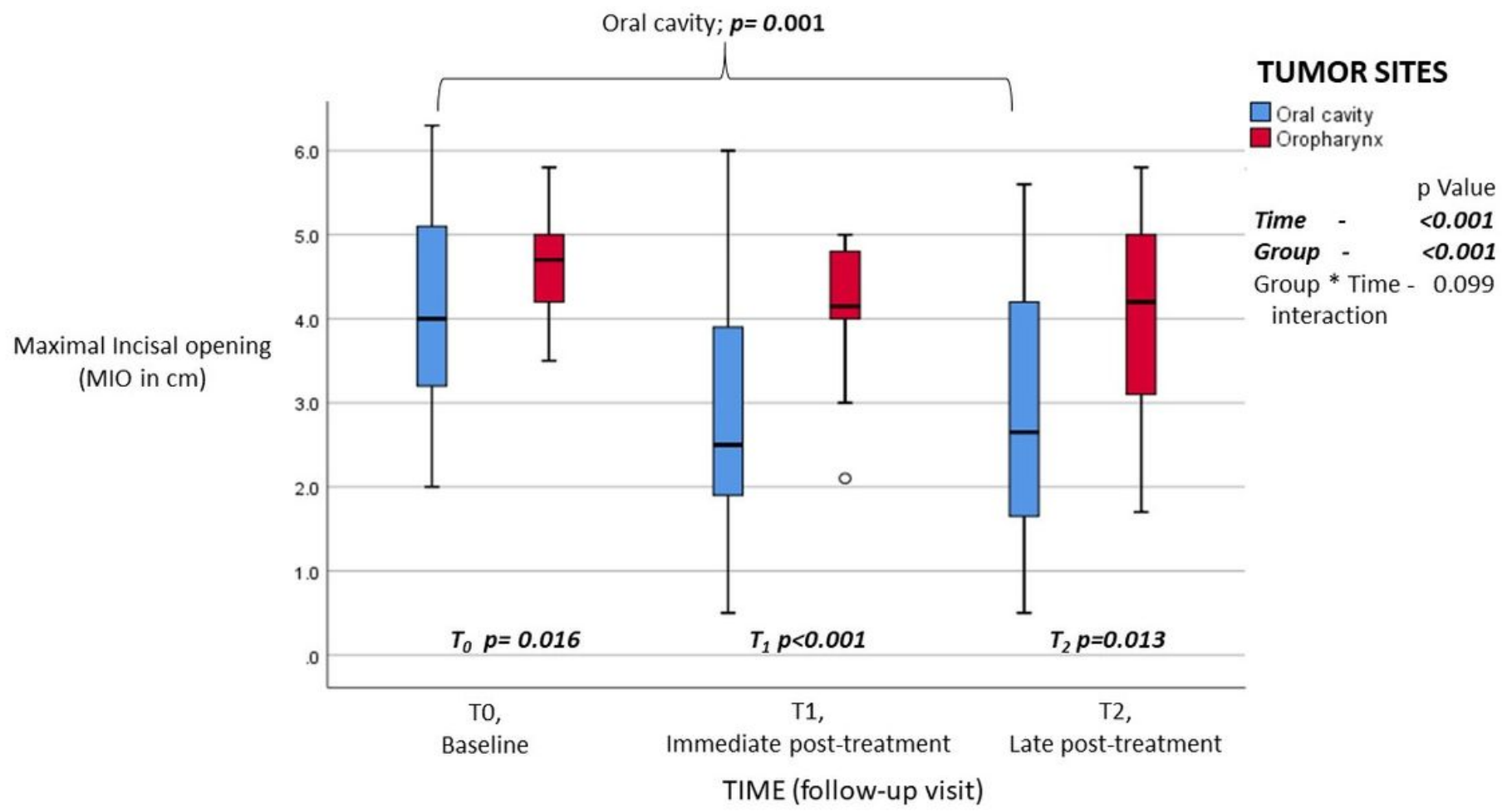

\section{Figure 1}

Results of non-parametric repeated measures ANOVA used to analyze longitudinal change in maximal incisal opening (cm) from baseline (T0) to 6-months post-treatment (T2), amongst patients stratified by tumor site, namely, oral cavity and oropharynx $(n=58)$. Inter-group pair-wise comparison at each time-point was done using independent T test or Mann-Whitney U test. Intra-group pair-wise analysis between baseline (T0) and 6-months post-treatment (T2) visits was done using Wilcoxon-sign ranked test. Differences with a p-value $<0.05$ considered to be statistically significant. 


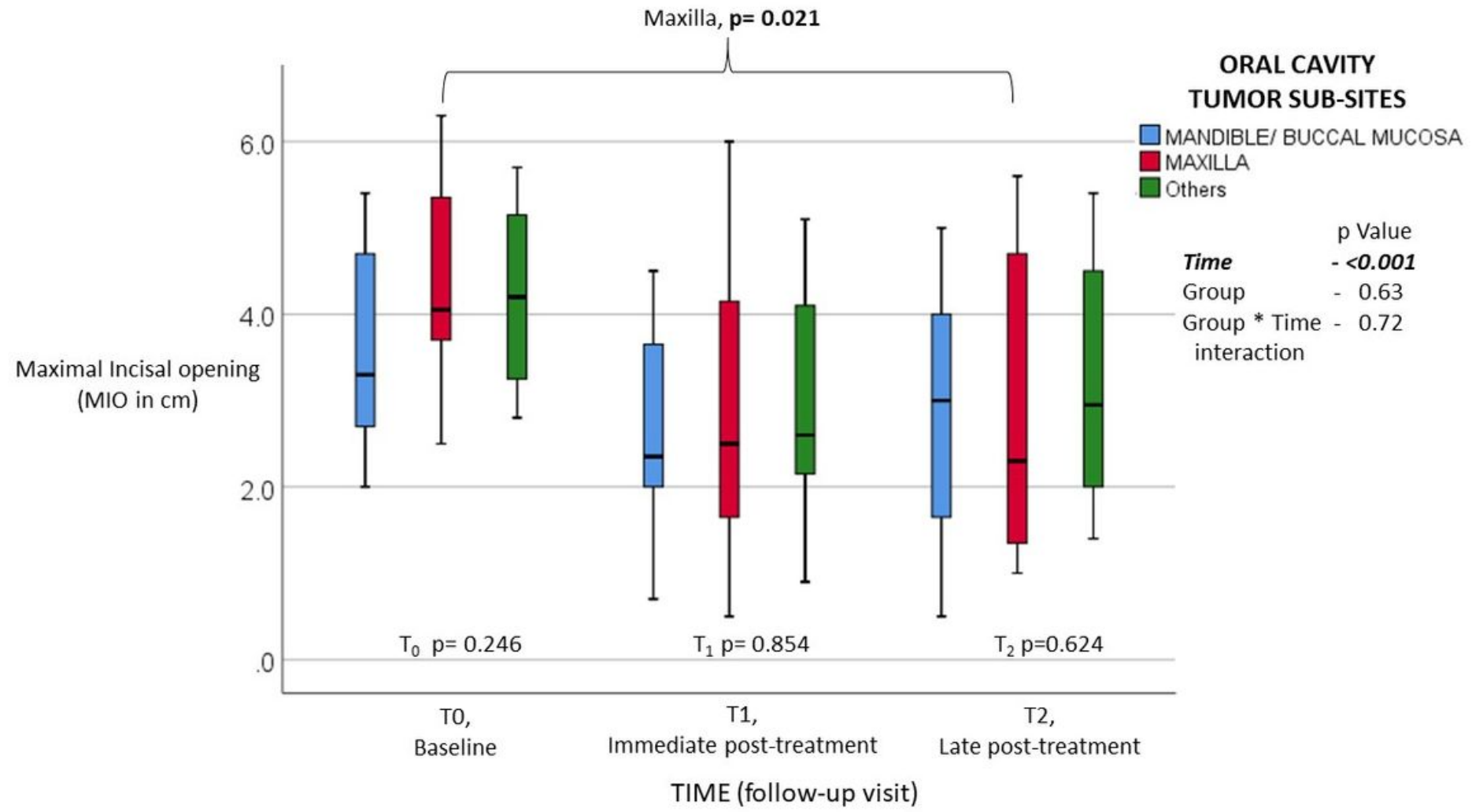

\section{Figure 2}

Results of non-parametric repeated measures ANOVA used to analyze longitudinal change in maximal incisal opening (cm) from baseline (T0) to 6-months post-treatment (T2), amongst patients stratified by oral cavity tumor sub-sites, namely, buccal mucosa-mandibular lesions; maxillary lesions; and midline (others) lesions $(n=58)$. Inter-group pair-wise comparison at each time-point was done using ANOVA or Kruskal Wallis test. Intragroup pair-wise analysis between baseline (T0) and 6-months post-treatment (T2) visits was done using Wilcoxon-sign ranked test. Differences with a p-value $<0.05$ considered to be statistically significant. 


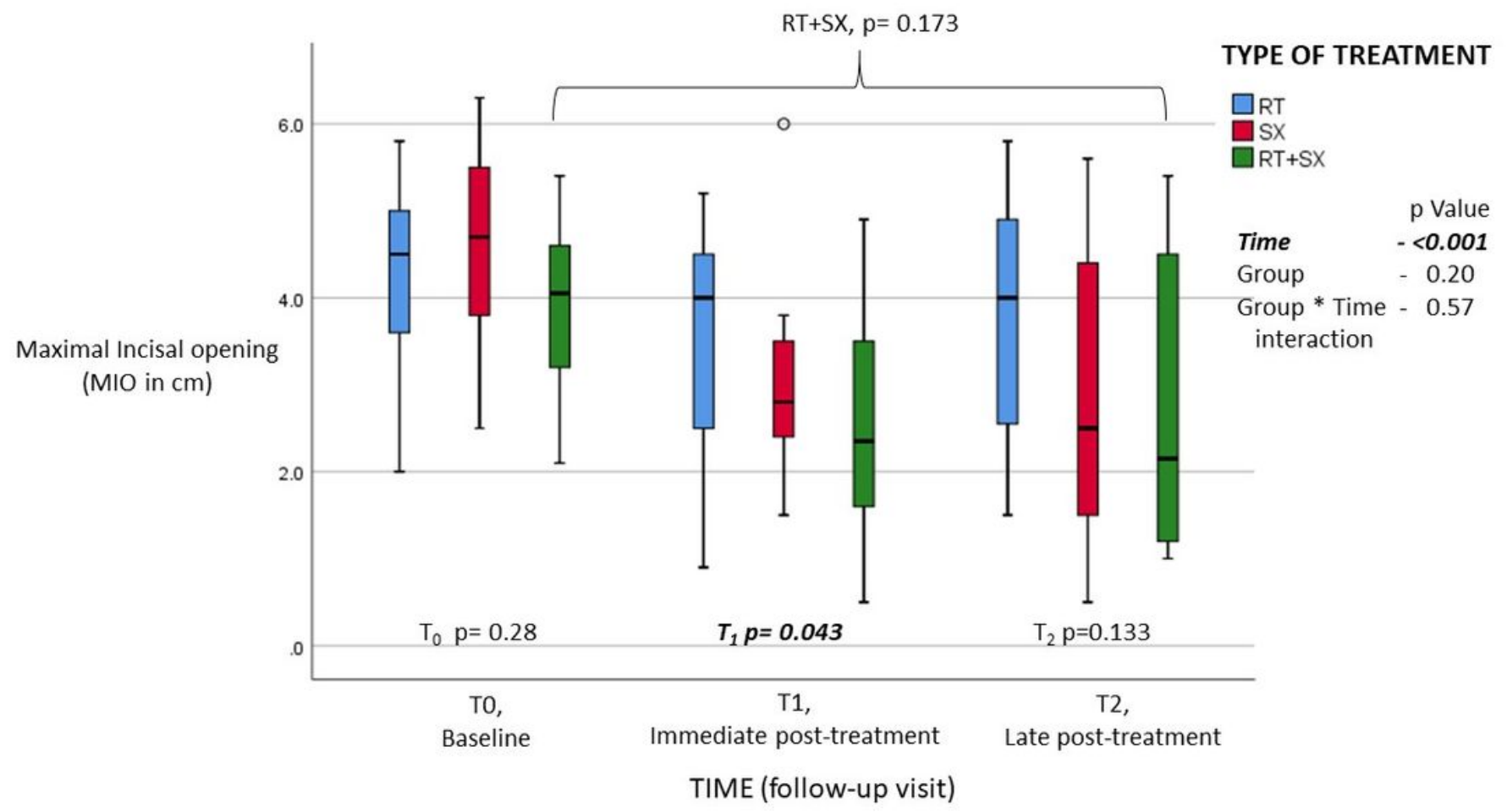

\section{Figure 3}

Results of non-parametric repeated measures ANOVA used to analyze longitudinal change in maximal incisal opening (cm) from baseline (T0) to 6-months post-treatment (T2), amongst patients stratified by treatment modalities, namely, surgery alone (SX); surgery + radiotherapy (RT+SX); and radiotherapy alone $(R T)(n=58)$. Inter-group pair-wise comparison at each time-point was done using ANOVA or Kruskal Wallis test. Intra-group pair-wise analysis between baseline (T0) and 6-months post-treatment (T2) visits was done using Wilcoxon-sign ranked test. Differences with a p-value $<0.05$ considered to be statistically significant.

\section{Supplementary Files}

This is a list of supplementary files associated with this preprint. Click to download.

- coidisclosureGPS.docx

- coidisclosureJD.docx

- coidisclosureJPA.docx

- coidisclosureKPD.docx

- coidisclosurePK.docx

- coidisclosurePR.docx

- coidisclosurePS.docx 